\title{
Elaboração de instrumento para padronização da passagem de plantão no Centro de Material e Esterilização
}

\author{
Standardization of the shift change at the Material and Sterilization Center \\ Estandarización de cambios de turno en el Centro de Material y Esterilización \\ Éderson Rodrigo Alves da Silva1*, Mara Lourenço Vermieiro.
}

\begin{abstract}
RESUMO
Objetivo: Padronizar a passagem de plantão no Centro de Material e Esterilização (CME) através da elaboração coletiva de um instrumento de qualidade. Métodos: Trata-se de uma pesquisa convergente assistencial, a qual valoriza a prática assistencial com fundamentação científica, compreendida em 5 fases: Concepção, Instrumentação, Perscrutação, Análise e Interpretação. Resultados: Os resultados foram conforme as fases metodológicas. Concepção: houve a fundamentação teórica da pesquisa, a qual proporcionou a construção do conhecimento e evidenciou a ausência de estudo desta temática no CME. A fase de Instrumentação constituiu no planejamento para obtenção de Informações e motivação dos colaboradores. Na fase de Perscrutação, houve a troca de saberes, construção do instrumento e educação permanente sobre a passagem de plantão. A fase de Análise ocorreu em meio a todas as fases, de modo a promover a reflexão, aplicação e validação do instrumento, conforme a realidade de cada período de trabalho. A última fase, interpretação, proporcionou a implementação e validação da aplicabilidade do instrumento, bem como sua normatização institucional. Conclusão: A padronização da passagem de plantão no CME é imprescindível para manutenção da continuidade e direcionamento do processo de trabalho no setor.
\end{abstract}

Palavras-chave: Comunicação em saúde, Equipe de enfermagem, Esterilização, Jornada de trabalho em turnos.

\section{ABSTRACT}

Objective: Standardize the shift change at the Material and Sterilization Center (MSC) through the collective elaboration of a quality instrument. Methods: This is a convergent care research, which values care practice with a scientific basis, comprising 5 phases: Conception, Instrumentation, Survey, Analysis and Interpretation. Results: The results were according to the methodological phases. Conception: there was the theoretical foundation of the research, which provided the construction of knowledge and evidenced the lack of study of this theme in the CME. The Instrumentation phase consisted of planning to obtain information and motivate employees. In the scrutiny phase, there was an exchange of knowledge, construction of the instrument and permanent education about the shift change. The Analysis phase took place in the middle of all phases, in order to promote reflection, application and validation of the instrument, according to the reality of each work period. The last phase, interpretation, provided the implementation and validation of the instrument's applicability, as well as its institutional standardization. Conclusion: The standardization of shift change at the $\mathrm{CME}$ is essential to maintain the continuity and direction of the work process in the sector.

Keywords: Communication in health, Nursing team, Sterilization, Shift work schedule.

\section{RESUMEN}

Objetivo: Estandarizar el cambio de turno en el Centro de Material y Esterilización (CME) mediante la elaboración colectiva de un instrumento de calidad. Métodos: Se trata de una investigación asistencial convergente, que valora la práctica asistencial con base científica, que comprende 5 fases: Concepción, Instrumentación, Relevamiento, Análisis e Interpretación. Resultados: Los resultados fueron de acuerdo a las fases metodológicas. Concepción: existió el fundamento teórico de la investigación, que brindó la

\footnotetext{
${ }^{1}$ Hospital Universitário da Universidade Federal da Grande Dourados (UFGD), Dourados - MS.

*E-mail: ederson.silva@ebserh.gov.br
} 
construcción del conocimiento y evidenció la falta de estudio de este tema en el CME. La fase de Instrumentación consistió en planificar para obtener información y motivar a los empleados. En la fase de escrutinio, hubo intercambio de conocimientos, construcción del instrumento y educación permanente sobre el cambio de turno. La fase de Análisis se desarrolló en medio de todas las fases, con el fin de promover la reflexión, aplicación y validación del instrumento, de acuerdo con la realidad de cada período de trabajo. La última fase, interpretación, brindó la implementación y validación de la aplicabilidad del instrumento, así como su estandarización institucional. Conclusión: La estandarización del cambio de turno en el CME es fundamental para mantener la continuidad y dirección del proceso de trabajo en el sector.

Palabras clave: Comunicación en salud, Equipo de enfermería, Esterilización, Horario de trabajo por turnos.

\section{INTRODUÇÃO}

A enfermagem desde sua criação possui o cuidado ao ser humano uma atividade fundamental do seu exercício profissional e, para que este cuidado seja realizado com qualidade, é imprescindível a utilização de recursos como os instrumentos nos processos de trabalho, os quais possibilitem a articulação da teoria com a prática (FELIPE TRL e SPIRI WC, 2019). Somado a isso, a comunicação envolvida no processo da passagem de plantão é outra premissa a ser considerada, uma vez que, por mais direcionada que seja 0 processo através de um instrumento padronizado, o repasse das informações deve acontecer de forma clara, objetiva e esclarecedora, uma vez que a comunicação de qualidade está diretamente ligada à continuidade das atividades de enfermagem e reflete na segurança do paciente (SILVA MF, et al., 2016).

Nesse contexto, não se pode negar que, dentre as atividades imprescindíveis para o exercício das atividades de Enfermagem, encontra-se a passagem de plantão com qualidade, já que nesse momento se pode repassar informações específicas de um paciente entre profissionais, entre equipes ou até mesmo aos familiares durante a visita, sendo essa atividade uma responsabilidade da equipe para com o paciente, em especial, do enfermeiro em assegurar a continuidade dos cuidados e segurança não apenas aos pacientes, mas também aos profissionais que irão manter suas ações voltadas ao paciente integrado num contexto ambiental (GONÇALVES MI, et al., 2017).

A comunicação é algo tão importante que a Organização Mundial da Saúde definiu como meta a melhoria da comunicação durante as passagens de plantões, seja ela pela forma verbalizada, escrita, em reuniões ou um mesclado de tudo isso (CORPOLATO RC, et al., 2019). Entretanto, levantamentos de estudos relacionados a passagem de plantão evidenciam que a verbalização direcionada de um instrumento escrito e padronizado é a forma mais utilizada, provendo maior efetividade na comunicação e menor risco de esquecimentos (CORPOLATO RC, et al., 2019; OLIVEIRA MC e ROCHA RGM, 2016).

Acontece que, embora existam grande proporção de artigos publicados relacionados à passagem de plantão na área da enfermagem, pouco se sabe no meio científico sobre a passagem de plantão no Centro de Material e Esterilização (CME). A situação fica ainda mais caótica quando se busca nas bases de dados um instrumento que oriente a passagem de plantão para a garantia de um do processo de trabalho de enfermagem voltado para a cultura de segurança através da comunicação entre turnos nas equipes que desenvolvem suas atividades no CME (SILVA EE e CAMPOS LF, 2007).

Nesse sentido, faz-se mister ressaltar a importância do setor no qual se processa os produtos de saúde utilizados na assistência direta ao paciente. Setor este, no qual recebe demanda de serviço de praticamente todos os setores de um hospital, com os mais variados materiais e relacionando com os mais diversos perfis profissionais. Isso reforça a necessidade de profissionais capacitados para dominar todas as particularidades em que o serviço venha a requerer e, para, além disso, dominar a tecnologia leve, qual seja, a comunicação durante a passagem de plantão (BUGSTV, et al., 2017).

Somado a isso, a comunicação a ser desenvolvida no CME deve ser com maior acurácia e por profissionais devidamente instruídos no assunto, para se evitar transtornos de continuidade da assistência, uma vez que os cuidados diretos aos pacientes dependem dos materiais produzidos e reprocessados nesse local. Ademais, a passagem de plantão com a comunicação mais efetiva é aquela com subsídio de um instrumento 
com informações escritas, então, a elaboração de um instrumento de passagem de plantão atenderá não apenas ao estabelecido pela Organização Mundial da Saúde (OMS), mas também ao proposto pelos estudos recentes dos quais tratam da qualidade da comunicação nas passagens de plantões (SILVA MF, et al., 2016; BUENO BRM, et al., 2015; LIMA AM e SILVA ER, 2017).

Nessa perspectiva, de avanços e promoção da qualidade e continuidade dos serviços de enfermagem, a passagem de plantão está intimamente ligada ao êxito neste processo. Desse modo, faz-se mister a sua execução com cientificidade e aplicabilidade, de modo não apenas a otimizar o tempo, mas também para a diminuição dos ruídos na comunicação e o estabelecimento do seu registro com clareza, sendo a padronização, através do uso de um instrumento uniformizado, uma excelente estratégia na gestão deste processo, uma vez que proporciona ao estabelecimento de saúde a continuidade das atividades, a melhoria na relação interpessoal, a qualificação das informações essenciais e a redução dos eventos adversos e suas consequências onerosas ao hospital público (MARQUES LF, et al., 2012; NASCIMENTO JSG, et al., 2018; PERUZZI LM, et al., 2019) .

Sendo assim, este estudo tem como objetivo padronizar a passagem de plantão em um CME através da elaboração com a participação e discussão coletiva de um instrumento de qualidade, validado e institucionalizado, uma vez que o local do presente estudo, comporta não apenas a equipe de enfermagem fixa do setor, como também outros colaboradores que realizam cobertura no setor e também os membros da equipe médica, os quais, por muitas vezes, possuem demanda peculiar em relação aos produtos de saúde segundo sua especialidade. Portanto, com a elaboração do instrumento, pretende-se promover uma comunicação efetiva com reflexo na qualidade e segurança do paciente.

\section{MÉTODOS}

Trata-se de um estudo qualitativo convergente assistencial, no qual valoriza a prática assistencial e compreende-se nas seguintes fases: a concepção, a instrumentação, a perscrutação, a análise e a interpretação, as quais foram realizadas no período de janeiro a junho de 2021, em um Hospital Universitário de Dourados. Houve a participação de 20 técnicos de enfermagem de um total de 22, sendo que 2 estavam de licença saúde e não puderam participar da pesquisa, os quais possuem experiência no setor há mais de 2 anos de atuação. A participação dos enfermeiros contou com 4 profissionais fixos do $\mathrm{CME}, 1$ enfermeiro do Centro Cirúrgico, o qual realiza cobertura recorrente no setor, e 1 enfermeira responsável Técnica do setor, todos possuem no mínimo uma especialização e um deles possui doutorado, dentre os quais, apenas 1 possui menos de 4 anos em Centro de Material e Esterilização e Centro Cirúrgico.

A pesquisa aconteceu em momento de pandemia, sendo respeitadas todas as normas de biossegurança e as datas estabelecidas para execução da Pesquisa Convergente Assistencial (PCA) segundo o mês do ano de 2021, conforme o cronograma abaixo (Quadro 1).

Quadro 1 - Cronograma de execução da Pesquisa no 1ํ semestre de 2021.

\begin{tabular}{|c|c|c|c|c|c|c|}
\hline ANO 2021 & Jan & Fev & Mar & Abr & Mai & Jun \\
\hline Fundamentação teórica & $\mathbf{X}$ & $\mathbf{X}$ & & & & \\
\hline Construção do projeto & $\mathbf{X}$ & $\mathbf{X}$ & & & & \\
\hline $\begin{array}{c}\text { Aprovação no Comitê de } \\
\text { Ética em Pesquisa }\end{array}$ & & & $\mathbf{X}$ & & & \\
\hline Reuniões & & & $\mathbf{X}$ & $\mathbf{X}$ & & \\
\hline Elaboração Instrumento & & & & $\mathbf{X}$ & $\mathbf{X}$ & \\
\hline Discussão/resultados & & & & & $\mathbf{X}$ & \\
\hline Finalização & & & & & $\mathbf{X}$ & $\mathbf{X}$ \\
\hline
\end{tabular}

Fonte: Silva ERA e Vermieiro ML, 2021. 
A PCA é um método que consegue evidenciar os processos de trabalho de enfermagem, contribuindo para a construção de estudos os quais unem as atividades assistenciais com a enfermagem baseada em evidências científicas. Além de ser uma pesquisa de fácil aplicabilidade no ambiente de trabalho, possui rigor metodológico em todo o seu processo de construção, além deste método estar intimamente relacionado a assistência de enfermagem e a gestão em saúde (PAIM L, et al., 2008; ROCHA PK, et al., 2012).

A Concepção do estudo, certamente, foi a primeira fase a ser construída, após uma revisão de literatura para o embasamento e enriquecimento das discussões em grupos. Após isso, verificou-se que os problemas enfrentados no processo assistencial, necessitaria de uma intervenção pelos autores inseridos no serviço, sendo desse modo, desenvolvida a pesquisa no Centro de Material e Esterilização de um hospital público da cidade de Dourados-MS. Isso foi possível devido a PCA, a qual converge prática diária com ações de inovações e melhorias a serem implementadas no contexto assistencial (THIOLLENT M, 2011).

O embasamento teórico para o trabalho foi realizado através de pesquisa em bases de dados MEDILINE, BDENF e LILACS. A pesquisa fora realizada de janeiro a fevereiro de 2021 para subsídio na fundamentação deste projeto. Foram utilizados como critérios de inclusão artigos publicados nos últimos 15 anos devido à escassez de estudos com a temática, também houve a inclusão daqueles que disponibilizassem de texto completo e no idioma português, segundo os Descritores em Saúde: Comunicação em saúde, Equipe de Enfermagem, Esterilização e Jornada de Trabalho em Turnos.

A segunda fase, Instrumentação, a qual tem como finalidade guiar os procedimentos metodológicos, incluindo: a escolha do campo de ação, que no caso desta pesquisa fora a comunicação em saúde no CME, sendo escolhidos os enfermeiros e técnicos de enfermagem que trabalham no setor. Esta escolha aconteceu guiada pelo embasamento da revisão de literatura, uma vez que muitos estudos não levaram em consideração a participação dos técnicos de enfermagem, desse modo, foram convidados a participarem todos os técnicos e enfermeiros do CME, exceto aqueles que se encontravam em licença saúde ou férias.

Durante este período, os pesquisadores definiram e captaram os recursos para realização de um diagnóstico situacional, através da consulta nos livros de registros, discussões nos grupos de aplicativos, fragilidades e potencialidades vivenciadas no setor para uma boa passagem de plantão.

A Terceira fase, Perscrutação, houve a definição das estratégias e suas execuções, para obtenção das informações importantes e elaboração do instrumento de passagem de plantão. Dentro desta fase, compreenderam-se duas etapas, as quais se convergiram, sendo o diagnóstico situacional e reuniões com grupos focais, de modo a permitir um ambiente menos formalista e promover a livre expressão de ideias sobre passagem de plantão, com a troca entre saberes dos pesquisadores e participantes envolvidos na pesquisa.

Na quarta fase, a Análise, ocorreu de modo a permear todas as fases da pesquisa, no entanto, esta fase foi de grande relevância ao selecionar as ideias e discussões trazidas até o momento para transformá-las em afirmativas em um check-list, o qual atendesse as especificidades de cada turno de trabalho. Nesse contexto, a revisão de cada ação durante todas as etapas pretendia proporcionar a construção de um instrumento bastante aplicável na prática, além de fácil e rápido preenchimento, entretanto, para que isso fosse realmente comprovado, a sua aplicabilidade deveria ser validada pelos profissionais envolvidos

Desse modo, a reunião com os grupos focais - por turno de trabalho - permitiu que os profissionais sentissem confortáveis para discorrer sobre o tema com troca de saberes entre técnicos de enfermagem, enfermeiros e pesquisadores. Embora as reuniões tenham acontecido nos períodos de trabalhos dos profissionais e entre colegas de turnos, por diversos momentos, houve a escuta dos participantes sobre a inclusão ou exclusão de ideias para a finalização do instrumento. Nesta fase da pesquisa, o instrumento já contemplava um apanhado de sugestões e sua estruturação já estava praticamente definida.

A quinta e última fase, a Interpretação, realizou-se a transcrição das informações obtidas durante a pesquisa, bem como a sua incorporação para a discussão dos seus resultados. Nesta fase, também está contemplada a validação do instrumento pelos enfermeiros e técnicos de enfermagem através da sua aplicação por duas semanas em todos os períodos no setor, com o registro comum ao enfermeiro e técnico de enfermagem, sendo o primeiro, responsável pela validação e complementação dos registros do técnico de 
enfermagem, para que o técnico da sala de preparo de materiais realize a assinatura no final, juntamente com enfermeiro, para ambos promoverem uma passagem de plantão com informações comuns a todos os membros da equipe anterior para a equipe do plantão seguinte.

As reuniões e discussões aconteceram de modo presencial em pequenos grupos, para atender não apenas o determinado pela OMS sobre o distanciamento social, como para conseguir ouvir com qualidade as demandas de todos os colaboradores que aceitaram a participar da PCA. Sendo assim, o instrumento foi elaborado e validado por todos os participantes envolvidos, uma vez que todos possuem expertise e vivência no $\mathrm{CME}$, conforme propõe a metodologia de pesquisa executada.

O instrumento padronizado foi pautado nos principais problemas levantados durante a passagem de plantão, contendo elementos relacionados à produtividade, organização, continuidade, supervisão e controle das atividades de enfermagem mais recorrentes e imprescindíveis ao processo do trabalho no CME, além da sua normatização em documento institucional como o Procedimento Operacional Padrão (POP).

Portanto, a PCA foi um estímulo ao envolvimento na pesquisa e seus desfechos, sendo levado em consideração todos os preceitos éticos e legais relacionados a aprovação no comitê de ética em pesquisa, a qual foi aprovada pelo Comitê de Ética em Pesquisa do Hospital Universitário da Grande Dourados através do Processo no 23529.002512/2021-90, contendo o Parecer no 13.2021, sendo todos os profissionais envolvidos - Técnicos e Enfermeiros do Centro de Material e Esterilização, orientados sobre a pesquisa e assinaram um Termo de Consentimento Livre e Esclarecido.

\section{RESULTADOS E DISCUSSÃO}

A realização desta pesquisa, por se tratar de método executado no processo de trabalho assistencial, apresenta como resultados, os desfechos das etapas compreendidas em cinco momentos: Concepção, Instrumentação, Perscrutação, Análise e Interpretação, nas quais, em cada uma delas, houve a promoção de grandes avanços no serviço em que os participantes e pesquisadores estão inseridos, uma vez que esta pesquisa é para contribuição de melhorias ao processo de trabalho do Centro de Material e Esterilização, com reflexo ainda maior, na segurança do paciente, na qualidade do serviço prestado e nas produção de conhecimento científico de qualidade (Quadro 2).

Quadro 2 - Resultados alcançados conforme execução das etapas da pesquisa

\begin{tabular}{|c|c|}
\hline Etapa da Pesquisa & Resultados \\
\hline Concepção & Embasamento das discussões, Construção do conhecimento. \\
\hline Instrumentação & $\begin{array}{c}\text { Motivação dos colaboradores, Obtenção de Informações no Diagnóstico } \\
\text { Situacional. }\end{array}$ \\
\hline Perscrutação & Troca de Saberes, Construção do Instrumento e Educação Permanente. \\
\hline Análise & Reflexão, Aplicação e Validação do Instrumento. \\
\hline Interpretação & $\begin{array}{c}\text { Discussão e Registro da Pesquisa, Normatização do Instrumento e Finalização e } \\
\text { Publicação da Pesquisa. }\end{array}$ \\
\hline
\end{tabular}

Fonte: Silva ERA e Vermieiro ML, 2021.

A revisão de literatura possibilitou o embasamento e enriquecimento das discussões nos grupos sobre a importância da comunicação efetiva, reforçando a necessidade da elaboração dos estudos relacionados a passagem de plantão, em especial, o CME, o qual é considerado um setor de encontro da assistência e gerenciamento, os quais demandam a articulação do domínio da comunicação em equipe e de tecnologias dos equipamentos (CAVALCANTE FML e BARROS LM, 2020; FASSARELLA CS, et al., 2021).

A definição dos profissionais envolvidos como participantes da pesquisa aconteceu após a revisão de literatura, uma vez que alguns trabalhos trouxeram a relevância da inclusão do técnico de enfermagem na 
construção de instrumentos assistenciais e gerenciais (CORPOLATO RC, et al., 2019). Frente a isso, os colaboradores se motivaram em fazer parte da pesquisa, tanto pelo interesse no assunto, como pela abordagem esclarecedora sobre a fundamentação teórica no momento do convite.

A captação de recursos e registro fomentou a obtenção de informações, sendo isso, uma competência gerencial, na qual o enfermeiro possui a habilidade de extração de dados nos meios de registros e experiência vivenciadas, através do Diagnóstico Situacional. Essa etapa, só foi possível com a atuação do papel do enfermeiro gerencial, o qual fundamenta a sua tomada de decisões de maneira planejada e subsidiada por dados e materialidade documental (CAVALCANTE FML e BARROS LM, 2020; SIMAN AG e BRITO MJM, 2016).

Nesta pesquisa, os problemas levantados foram: a necessidade de registro sobre produtividade, controle de estoque, supervisão do trabalho e indicadores de atividades no setor, eixos estes, trabalhados na construção do instrumento. Desse modo, as informações colhidas no diagnóstico situacional, não só serviram de start para as discussões em grupo, como fomentaram as trocas de saberes conforme o instrumento estava sendo esboçado, sendo o enfermeiro, o responsável por despertar do senso crítico da sua equipe, de maneira coordenada, para o comprometimento da equipe no processo de trabalho através da reflexão e correção de práticas inadequadas (SIMAN AG e BRITO MJM, 2016).

Somado a isso, o encontro com ampla discussão da forma de executar as atividades no setor, permitiu que, naturalmente, acontecesse a educação permanente de todos os envolvidos, a partir do momento em que trouxe mudanças profundas na prática diária do serviço, refletindo na segurança do paciente e no comprometimento das tarefas executadas (SILVA PSC, et al, 2013; ATHANÁZIO AR e CORDEIRO BC, 2015).

"[...] A discussão sobre passagem de plantão em um instrumento, proporciona reconhecimento da nossa experiência e nos permite aprender e contribuir com o nosso trabalho, além da segurança pessoal e ao paciente, pois o instrumento é de acordo com a nossa realidade e não algo imposto" (P1).

Nessa perspectiva, conforme os profissionais traziam suas percepções acerca do tema e, conforme o diálogo tomava proporções específicas da equipe e turno do grupo focal, as afirmativas e perguntas do instrumento padronizado também tomava sua configuração peculiar aplicado em cada turno de trabalho, indo ao encontro do que defendo Lino QR (2019), a utilização de instrumentos os quais facilitem a comunicação entre equipes, com clareza, objetividade e focado na qualidade da assistência e segurança do paciente.

$O$ instrumento foi dividido em três partes, duas nas quais contemplam os períodos matutino e vespertino e outra destinada ao noturno. Foram criadas afirmativas nas quais pudessem ser marcadas com um " $X$ " 0 campo correspondente a condição realizada no setor ou descrever sucintamente algumas informações em pequenos espaços. No instrumento contêm informações relacionadas desde execuções de atividades mais técnicas, até as atividades mais gerenciais, além da destinação de um espaço de observações para relatório de questões menos recorrentes, intercorrências ou não contempladas no check-list, as quais fogem à rotina (Dados Suplementar).

O reconhecimento, validação e implementação do instrumento pelos enfermeiros e técnicos de enfermagem permitiu que tal sistematização fosse legitimada e direcionada para normatização institucional, garantindo assim, que o instrumento fosse executado no CME e direcionado por um documento instrutor, a saber o POP (FELIPE TRL e SPIRI WC, 2019).

Nessa mesma perspectiva, a implementação de um instrumento padronizado elaborado, permite que o enfermeiro quantifique as ações positivas, utilize como banco de dados para fundamentação do seu gerenciamento de materiais, no controle de produção, esterilização e dispensação, norteado por recursos documentais e institucionais, para que seja algo facilitador no tratamento desses de dados, para a sua utilização nas tomadas de decisões e mudança ou manutenção dos processos de acordo com o que se deseja alcançar, não apenas naquilo que é importante ao setor, mas também no que se interessa a instituição como um todo (CAVALCANTE FML e BARROS LM, 2020; MARQUES LF, et al., 2012; PERUZZI LM, et al., 2019). 
Nesse sentido, em concordância ao que Beccaria LM, et al. (2017), não se pode negar a real importância e necessidade do papel do enfermeiro e sua equipe no processo gerencial e assistencial, sendo a passagem de plantão a maior ferramenta que garante a manutenção de tudo que se construiu de positivo no trabalho. Entretanto, este repasse de informações deve ser efetivo, através de profissionais dotados de habilidades, seja pelo método de registro ou verbalizado. Atrelado a isso, Barbosa PMK, et al. (2013) salienta que a utilização de um instrumento padronizado, o qual venha a direcionar no processo de trabalho, não apenas facilita a comunicação, mas contribui com a cultura de segurança do paciente e do ambiente de trabalho.

Sendo assim, o instrumento construído demostrou-se importante, haja vista ser um meio de comunicação no qual evita perda da qualidade dos cuidados e diminui riscos na segurança do paciente relacionados a continuidades das atividades entre turnos, pois a passagem de plantão padronizada otimiza o tempo gasto entre turnos, reduz ruídos e serve como consulta em caso de esquecimento daquilo que foi repassado verbalmente (SETTANI SS, et al., 2019).

Portanto, a construção de um instrumento, no qual registra-se a comunicação, é defendida por diversos autores que possuem expertise no assunto. Para tanto, o instrumento deve ser elaborado com a devida aplicabilidade e aceitação do grupo, como foi o exemplo desta pesquisa, uma vez que não apenas 0 instrumento fora apenas testado pela equipe, como validado por profissionais com experiência no setor (ANDRADE WV, et al., 2019).

Outro fator importante, foi a elaboração de um documento institucional no qual viesse a discorrer sobre a passagem de plantão no instrumento padronizado, o Procedimento Operacional padrão - POP, etapa esta, também executada e enfatizada por Corpolato RC, et al. (2019) o qual reforça o estabelecimento da implantação do POP através da sua publicação em documento institucional,o qual direciona sobre a passagem de plantão, tornando a prática da comunicação um meio qualificado, bem como pelo incentivo institucional para que os profissionais envolvidos contribuam com a política de segurança do paciente estabelecida pelo hospital.

Portanto, em consonância ao exposto até o momento, este instrumento alcançou seus objetivos por ter sido elaborado de forma coletiva, com qualidade, promovendo mudanças e quebras de paradigmas, legitimando-o através da implementação e validação pelos profissionais os quais possuem expertise no campo de atuação, além da sua normatização por um POP referente a passagem de plantão no CME.

\section{CONCLUSÃO}

Diante do exposto, a PCA permitiu que a passagem de plantão pudesse ser padronizada e discutida com os envolvidos no processo de trabalho, promovendo grandes resultados no ambiente de trabalho, em especial, a percepção da real importância da passagem de plantão com qualidade, a educação permanente com a troca de saberes entre os profissionais envolvidos e a produção de ciência com responsabilidade. Além disso, a construção e validação de um instrumento que atendesse a expectativa da equipe de um CME contribuiu para otimização do tempo, promoção da qualidade e supervisão das atividades de enfermagem. Nesse entendimento, o instrumento também serve de modelo para outras instituições, as quais podem adaptá-lo ou mesmo utilizá-lo na sua totalidade, pois não fora encontrado algo publicado na literatura levantada nos últimos 15 anos. Portanto, a padronização da passagem de plantão no CME é imprescindível para manutenção da continuidade e direcionamento do processo de trabalho no setor, contribuindo para uma comunicação mais efetiva entre equipe.

\section{REFERÊNCIAS}

1. ANDRADE WV, et al. Comunicação entre Gestores e Equipe das Unidades de Pronto Atendimento. Revista de Enfermagem UFPE online, 2019; 13: 1-5.

2. ATHANÁZIO AR; CORDEIRO BC. Educação Permanente a Trabalhadores da Central de Material e Esterilização. Rev enferm UFPE on line., 2015; 9(Supl. 6): 8758-61.

3. BARBOSA PMK, et al. Organização do processo de trabalho para passagem de plantão utilizando escore para dependência e risco clínico. Revista de Atenção à Saúde, 2013; 15(58): $19-26$. 
4. BUENO BRM, et al. Caracterização da Passagem de Plantão entre o Centro Cirúrgico e a Unidade de Terapia Intensiva. Revista Cogitare Enfermagem. Goiás, 2015; 20(3): 512-518.

5. BUGSTV. et al. Perfil da equipe de Enfermagem e percepções do trabalho realizado em uma central de materiais. REME - Rev Min Enferm., 2017; 1-8.

6. CAVALCANTE FML, BARROS LM. O trabalho do enfermeiro no Centro de Material e Esterilização: uma revisão integrativa. REV. SOBECC, SÃO PAULO, 2020; 25(3): 171-178.

7. CIAMPONE MHT; PERES AM. Gerência e Competências Gerenciais do Enfermeiro. Revista Texto e Contexto em Enfermagem, 2006; 15(3): 492-499.

8. CORPOLATO RC. et al. Padronização da passagem de plantão em Unidade de Terapia Intensiva Geral Adulto. Rev Bras Enferm [Internet]., 2019; 95-102.

9. FASSARELLA CS, et al. Produção de Enfermagem em Centro de Material e Esterilização: Um Estudo Bibliométrico. Rev Fun Care Online. 2021; 13: 829-835.

10.FELIPE TRL, SPIRI WC. Construção de um Instrumento de Passagem de Plantão. Revista Enfermagem em Foco, 2019; 76-82.

11.GONÇALVES MI et al. A segurança e passagem de plantão em unidades de cuidados intensivos neonatais. Rev. Baiana de Enfermagem, 2017; 31(2): 1-10.

12. LIMA AM, SILVAER. Sistematização da passagem de plantão em unidade de terapia intensiva: proposta de instrumento de registro. Revista Científica de Enfermagem, 2017; 7(20): 48-57.

13. LINO QR, et al. Resumo: Instrumento de Passagem de Plantão para Técnicos De Enfermagem de uma UTI de Hospital Privado de Curitiba Anais Do Evinci - UniBrasil, Curitiba, 2019; 5(1): 339-339.

14.MARQUES LF, et al. A passagem de plantão como elemento fundamental no processo de cuidar em enfermagem: 0 perfil da equipe de enfermagem de um hospital universitário. R. pesq.: cuid. fundam. Online, 2012; 4(2): $2878-82$.

15.NASCIMENTO JSJ, et al. Passagem de plantão como ferramenta de gestão para a segurança do paciente. Rev Enferm UFSM, 2018; 8(2): 544-559.

16. OLIVEIRA MC, ROCHA RGM. Reflexão acerca da passagem de plantão: implicações na continuidade da assistência de enfermagem. Enfermagem e Vista, 2016; 226-233.

17.PAIM L, et al. Pesquisa Convergente-Assistencial e sua Aplicação em Cenários da Enfermagem. Cogitare Enfermagem, Paraná, 2008; 13(3): 380-386.

18. PEREIRA, BT, et al. A Passagem de Plantão e a corrida de Leito como Instrumentos Norteadores para o Planejamento da Assistência de Enfermagem. remE - Rev. Min. Enferm., 2011; 15(2): 283-289.

19.PERUZZI, LM, et al. Passagem de Plantão na Atenção Hospitalar. Rev enferm UFPE on line, 2019; $13(4)$ : $989-996$.

20.ROCHA PK, et al. Pesquisa Convergente Assistencial: uso na elaboração de modelos de cuidado de enfermagem. Rev Bras Enferm., 2012; 65(6): 1019-25.

21.SCHORR V, et al. Passagem de plantão em um serviço hospitalar de emergência: perspectivas de uma equipe multiprofissional. Interface (Botucatu), 2020; 1-16.

22.SETTANI SS, et al. Comunicação de Enfermagem e as Repercussões na Segurança do Paciente. Revista de enfermagem da UFPE on line, 2019; 13: 1-7.

23. SILVA EE, CAMPOSLF. Passagem de Plantão na Enfermagem: Revisão da Literatura. Revista Cogitare Enfermagem, 2007; 12(4): 502-507.

24.SILVA MF, et al. Comunicação na Passagem de Plantão de Enfermagem: Segurança do Paciente Pediátrico. Revista Texto Contexto Enferm., 2016; 25(3): 1-10.

25.SILVA PSC, et al. Atuação da enfermagem na central de material e esterilização em um hospital de Teresina. Rev Interd., 2013; 6(3): 45-51.

26. SIMAN AG e BRITO MJM. Mudanças na prática de enfermagem para melhorar a segurança do paciente. Rev Gaúcha Enferm., 2016; 37: 1-9.

27.THIOLLENT M. Metodologia da Pesquisa Ação. 18 ed. São Paulo: Cortez, 2011. 136 p. 